\title{
Effect of inorganic salts and matrix crosslinking on the dose response of polymer gel dosimeters based on acrylamide
}

\author{
David Chacón $^{\mathrm{a}, \mathrm{b}}$, Miriam Strumia ${ }^{\mathrm{c}, \mathrm{d}}$, Mauro Valente ${ }^{\mathrm{a}, \mathrm{e}, \mathrm{f}}$, Facundo Mattea ${ }^{\mathrm{a}, \mathrm{c}, \mathrm{d}, *}$ \\ ${ }^{\text {a } U n i v e r s i d a d ~ N a c i o n a l ~ d e ~ C o ́ r d o b a, ~ F a c u l t a d ~ d e ~ M a t e m a ́ t i c a, ~ A s t r o n o m i ́ a, ~ F i ́ s i c a ~ y ~ C o m p u t a c i o ́ n, ~ L a b o r a t o r i o ~ d e ~ I n v e s t i g a c i o ́ n ~ e ~ I n s t r u m e n t a c i o ́ n ~ e n ~ F i ́ s i c a ~ A p l i c a d a ~ a ~ l a ~}$ \\ Medicina e Imágenes por Rayos X, LIIFAMIR ${ }^{\otimes}$, Córdoba, Argentina \\ b Departamento de Física, Universidad Nacional, Heredia, Costa Rica \\ ${ }^{\mathrm{c}}$ Universidad Nacional de Córdoba, Facultad de Ciencias Químicas, Departamento de Química Orgánica, Córdoba, Argentina \\ d Instituto de Investigación y Desarrollo en Ingeniería de Procesos y Química Aplicada, IPQA, CONICET, Córdoba, Argentina \\ e Instituto de Física E. Gaviola, IFEG, CONICET, Córdoba, Argentina \\ ${ }^{\mathrm{f}}$ Centro de Física e Ingeniería en Medicina, CFIM \& Departamento de Ciencias Físicas, Universidad de La Frontera, Temuco, Chile
}

\section{A R T I C L E I N F O}

\section{Keywords:}

Inorganic salts

Polymer

Dosimetry

Acrylamide

Gelatin crosslinking

\begin{abstract}
A B S T R A C T
The use of additives in polymer gel dosimeters to enhance their sensitivity or to improve their performance is of great importance for their application in radiomedicine. Inorganic salts have been used as additives with this purpose; however, their presence in the dosimeters induce severe modifications in their mechanical properties and consequently in their capabilities to maintain a stable dose distribution with time. Most studies in this area conclude in the need to use chemical modifications of the species responsible for the mechanical properties of the dosimeters, which in most cases is a gelatin matrix. In this study, a covalent crosslinking of the gelatin matrix of PAGAT dosimeters doped with different inorganic salts, namely $\mathrm{MgCl}_{2}, \mathrm{CaCl}_{2}$ and $\mathrm{MnCl}_{2}$ has been carried out. The mechanical properties, X-ray sensitivity and dose distribution stability in these materials were compared to those of PAGAT. The results indicated a compromise between the crosslinking of the gelatin structure and the sensitivity of the dosimetric material. Therefore, the proper selection of the degree of crosslinking and the inorganic salt concentration must be considered. An enhanced dosimeter was obtained by adding $\mathrm{MgCl}_{2}$ with a $1 M$ concentration and by crosslinking the gelatin matrix with glutaraldehyde at a $0.08 \% w / v$ concentration. This material presented a $75 \%$ enhanced sensitivity relative to PAGAT and similar temporal stability and spatial stability in 2D dose distributions.
\end{abstract}

\section{Introduction}

One of the main tasks for a medical physicist is to control the dose distribution in radiotherapy treatments and in patient radiation protection during diagnostic radiology. Gel dosimetry has the potential to be great tools in these applications, because unlike common dosimeters, such as ionization chambers, thermoluminescent dosimeters and films, they don't have any limitations to record and maintain spatial dose distribution. Additionally, gel dosimetry has the advantage of providing, not only quantitative information on the delivered dose, but also a three-dimensional dose distribution with high resolution (Ibbott, 2004). Among the different types of gel dosimeters, polymer gel dosimeters (PGDs) have been proposed and used in the last decades because of their low post-irradiation diffusion compared to other systems like Fricke Gel dosimetry (Baldock et al., 2010). Since 1993, when the first polymer gel dosimeter (PGD) was proposed (Maryanski et al., 1993), different types of monomers and compositions have been suggested and studied (Titus et al., 2016; Rabaeh et al., 2008) to obtain a dosimetric system with the optimal dose-response, temporal and spatial stability, energy and dose rate independency and with preparation methods simple enough to be used in clinical applications.

In the last five years, the use of inorganic salts to enhance the sensitivity of PGDs to radiation has been investigated. Initially, in 2012 Hayashi et al. (2012) showed that there was a clear effect on a methacrylic-acid-based PGD with the addition of some inorganic salts. They observed an increase in the R2-dose sensitivity when $\mathrm{MgCl}_{2}$ was incorporated in the PGD. In their results, it was found that the polymerization rate increased with the addition of inorganic salts by measuring temperature changes caused by the exothermic polymerization reactions during the irradiation. The authors suggested that this effect was correlated with the hydration properties of metal cations such as $\left(\mathrm{Li}^{+}, \mathrm{Na}^{+}, \mathrm{K}^{+}\right.$and $\mathrm{Mg}^{+2}$ ), which promotes the mobility and reactivity of

\footnotetext{
* Corresponding author. Universidad Nacional de Córdoba, Facultad de Ciencias Químicas, Departamento de Química Orgánica, Córdoba, Argentina.

E-mail addresses: fmattea@gmail.com, fmattea@fcq.unc.edu.ar (F. Mattea).
} 
the radicals involved in the polymerization reactions and also on their termination rate. Similar effects were reported from the same authors with a polyacrylamide based gel (PAGAT) (Hayashi et al., 2013), where an increase in the nuclear magnetic resonance (NMR) response of the dosimeters was reported. However, a significant depression of the gels melting point caused by the addition of inorganic salts represented a great drawback for the application of these materials (Al-Jarrah et al., 2016). They concluded that some type of crosslinking agent should be used in the gelatin matrix of the PGDs to avoid it. The melting point depression and changes on the mechanical properties of gelatin matrix have been reported for different applications and related to changes in the electrostatic properties of the gel (Haug et al., 2004; Sarabia et al., 2000).

The use of formaldehyde in different PGDs to increase their melting point has been studied by several authors (Fernandes et al., 2008; Aziz et al., 2013; Pavoni and Baffa, 2012) obtaining an increase in the melting point of about $40^{\circ} \mathrm{C}$. Recently, glutaraldehyde (GTA), a less toxic crosslinking agent than formaldehyde, has been studied (Romero et al., 2016) with the aim of improving the thermal stability of PGDs. This study showed that, if concentrations lower than $0.50 \% w / v$ of GTA were used to crosslink the gelatin matrix, its thermal stability was significantly improved and an increase of the elastic modulus up to 100 times was achieved at $37^{\circ} \mathrm{C}$. In addition, the analytical responses of crosslinked and unmodified itaconic acid based dosimeters (Mattea et al., 2015) were compared with no significant differences between each other.

The main goal of the present study is to evaluate the effect of inorganic salts and GTA on a polymer gel dosimeter based on acrylamide (PAGAT). For this purpose, the rheological characterization of different dosimetric systems with and without inorganic salts and GTA was carried out to evaluate the elastic properties of the material together with the dosimetric response to X-ray radiation. In this study, the ions selection was based on their ionic radius and on their effect on the structure of water according to Y. Marcus (2009). In this way, $\mathrm{MgCl}_{2}$, $\mathrm{MnCl}_{2}$ and $\mathrm{CaCl}_{2}$ were selected because of their higher capability on forming water structures as depicted in Fig. 1.

\section{Material and methods}

\subsection{PGD manufacturing}

Acrylamide based gels (PAGAT) were manufactured based on the method described by Venning et al. (2005) using $89 \% w / w$ of ultrapure deionized water, $5 \% w / w$ of gelatin (250 Bloom purchased from FLUKA), $3 \% w / w$ of N, N' methylene bisacrylamide (BIS, 99\% purchased from Sigma Aldrich ${ }^{\circ}$ ), $3 \% w / w$ of acrylamide (AAm, $99 \%$ purchased from

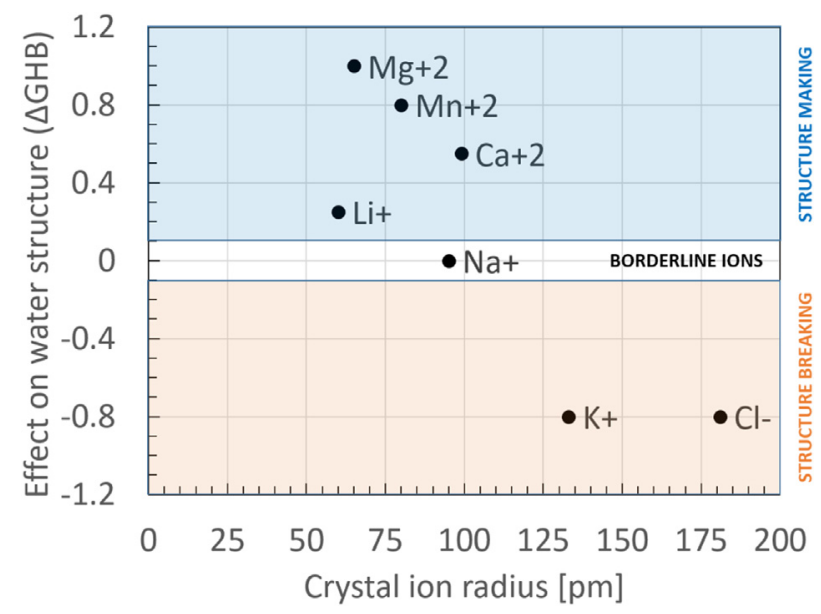

Fig. 1. a) Effect on the structure of water vs crystal ionic radius.
Sigma Aldrich ${ }^{\circledR}$ ) and $10 \mathrm{mM}$ of Tetrakis(hydroxymethyl)phosphonium chloride (THPC, 80\% solution purchased from FLUKA). Briefly, $90 \%$ of the water used in the dosimeters was mixed with the gelatin for $10 \mathrm{~min}$ at room temperature and stirred at $250 \mathrm{rpm}$. Afterwards, the temperature was set to $45^{\circ} \mathrm{C}$ with constant stirring until a homogenous solution was obtained. Then, BIS was incorporated and the whole solution was mixed for $15 \mathrm{~min}$ at $45^{\circ} \mathrm{C}$. Next, the temperature was lowered to $37^{\circ} \mathrm{C}$ and the AAm was incorporated. The whole solution was mixed at $37^{\circ} \mathrm{C}$ for $30 \mathrm{~min}$ and the THPC was added with the remaining $10 \%$ of the water at $35^{\circ} \mathrm{C}$. The obtained solution was kept at this condition for $2 \mathrm{~min}$. Two types of dosimeters were prepared with the obtained material, first PMMA cuvettes of $10 \times 5 \times 40 \mathrm{~mm}^{3}$ were filled with the sensitive material and used for the dosimetric response analysis, and then PMMA layer containers of $50 \times 50 \times 5 \mathrm{~mm}^{3}$ were prepared and used to study dose distribution characteristics in the dosimeters. Finally, the containers were sealed and stored at $4{ }^{\circ} \mathrm{C}$ until their irradiation. For materials containing inorganic salts, a $1 M$ solution of the specific salt $\left(\mathrm{MgCl}_{2}, \mathrm{CaCl}_{2}\right.$ or $\left.\mathrm{MnCl}_{2}\right)$ was used instead of water. Furthermore, for crosslinked gels, glutaraldehyde (GTA, 50\% $w / w$ purchased from Sigma Aldrich ${ }^{\circ}$ ) was incorporated after the THPC at the last minute of the preparation. Table 1 summarizes the different concentrations and materials used in this study. For the rheological characterization the dosimetric materials were placed in cylindrical containers suitable for the analysis.

\subsection{Irradiation}

The different sets were irradiated in an X-ray tube with a $W$ anode connected to a Siemens Kristalloflex generator with a maximum power of $3 \mathrm{~kW}$, described elsewhere (Valente et al., 2016). The electrical current range of the generator goes from 5 to $50 \mathrm{~mA}$ and the voltage range from 20 to $60 \mathrm{kVp}$. In the irradiation experiments the incident beam was collimated to a $5 \times 5 \mathrm{~cm}^{2}$ square geometry, using an electrical current of $44 \mathrm{~mA}$, voltage of $44 \mathrm{kVp}$, a source-to-phantom distance of $800 \mathrm{~mm}$ and a collimator to sample distance of $10 \mathrm{~mm}$. In order to improve absorbed dose uniformity, the cuvette dosimeters were irradiated with a "Box"-like technique using four opposite and parallel fields with a dose rate of $100 \mathrm{cGy} / \mathrm{min}$. All irradiations were carried out by triplicate and at a controlled temperature of $25^{\circ} \mathrm{C}$. For the layer type dosimeters, the irradiations were carried out with the same beam quality using a circular collimator with a diameter of $15 \mathrm{~mm}$ and a dose rate of $120 \mathrm{cGy} / \mathrm{min}$ at $25^{\circ} \mathrm{C}$. The delivered dose of each dosimeter was set to have similar responses in each material. Therefore, PAGAT dosimeters were irradiated with a dose of $10 \mathrm{~Gy}$, and materials containing inorganic salts, with or without GTA crosslinking, were irradiated with a dose of 5 Gy.

Table 1

PGDs used in this study.

\begin{tabular}{lllll}
\hline \multirow{2}{*}{ Set } & PGD & Inorganic Salt & GTA concentration & Dose Range \\
\cline { 3 - 5 } & & & $(\% w / v)$ & (Gy) \\
\hline P1 & PAGAT & - & - & $0-16$ \\
P2 & PAGATMg & $\mathrm{MgCl}_{2}$ & - & $0-10$ \\
P3 & PAGATCa & $\mathrm{CaCl}_{2}$ & - & $0-12$ \\
P4 & PAGATMn & $\mathrm{MnCl}_{2}$ & - & $0-12$ \\
P5 & PAGAT(Liq) & - & - & $0-12$ \\
G1 & PAGAT + GTA26 & - & 0.26 & $0-18$ \\
G2 & PAGAT + GTA15 & - & 0.15 & $0-18$ \\
G3 & PAGAT + GTA8 & - & 0.08 & 0.10 \\
PG1 & PAGATMg + GTA26 & $\mathrm{MgCl}_{2}$ & 0.26 & $0-18$ \\
PG2 & PAGATMg + GTA15 & $\mathrm{MgCl}_{2}$ & 0.15 & $0-15$ \\
PG3 & PAGATMg + GTA8 & $\mathrm{MgCl}_{2}$ & 0.08 & $0-10$ \\
\end{tabular}

a The PAGAT(Liq) was stored at $35^{\circ} \mathrm{C}$ until its irradiation to keep it in a liquid state. 


\subsection{Optical absorbance measurement}

A UNICO ${ }^{\circ}$ S1205 spectrophotometer was used to measure the optical absorbance of all irradiated samples. The absorbance of the PGDs was determined at $540 \mathrm{~nm}$ before and $24 \mathrm{~h}$ after their irradiation, which has been reported as the optimal stabilization period for the polymerization reactions within a PGD (Senden et al., 2006). A relative absorbance $(\Delta A)$ was defined as the difference between the optical absorbance of the irradiated sample $\left(A_{i}\right)$ and its corresponding nonirradiated sample $\left(A_{0}\right)$. Then, $\Delta A$ was fitted to a linear function of the dose $(D)$, where the slope $(s)$ represents the PGD sensitivity.

$\Delta A=A_{i}-A_{0}=s D+n$

Aiming on quantifying the effect of adding the different salts to the acrylamide based PGD, a sensitivity variation parameter $(S V)$ was defined as the ratio between the dose-response slopes with and without inorganic salts, as expressed in Equation (2)

$S V=\frac{S_{\text {Test }}-S_{\text {Ref }}}{S_{\text {Ref }}}$

Where $S_{\text {Test }}$ and $S_{\text {Ref }}$ are the fitted slopes for the modified materials and PAGAT, respectively.

Expanded uncertainties $(U)$ were calculated as a function of the number of samples and a coverage factor $(k)$ associated to a Student's tdistribution with a67\% confidence value. The expression used to calculate $U$ is presented in Equation (3), where $f$ is a function of $x_{i}$, and $u_{x_{i}}$ is the uncertainty in $x_{i}$ (Taylor, 1997).

$U=k \sqrt{\left(\sum_{i}\left(\frac{\partial f}{\partial x_{i}}\right)^{2} u_{x_{i}}^{2}\right)}$

The uncertainties in the dose response of each material were obtained by using Equation (3), where $\mathrm{U}$ represents the uncertainty in the slope (s) of $\Delta A$ vs dose, $f$ is the mean $s$ value for each material, $x_{i}$ are the $\Delta A$ values for each dose, and $u_{x_{i}}$ are the uncertainties in each $\Delta A$ value.

\subsection{Rheometry}

The elastic modulus $\left(G^{\prime}\right)$ of each dosimetric system was studied with an Anton Paar MCR 301 rheometer. Samples were stored at $4{ }^{\circ} \mathrm{C}$, with the exception of liquid PAGAT that was stored at $35^{\circ} \mathrm{C}$ until its analysis. Measurements were performed on circular sample holders of 8 and $50 \mathrm{~mm}$ diameter, depending on the $G^{\prime}$ value range. The sample gap was set to $2 \mathrm{~mm}$ for the $8 \mathrm{~mm}$ sample holder and $1 \mathrm{~mm}$ for the $50 \mathrm{~mm}$ sample holder. A closed chamber configuration was used to maintain the temperature at a desired value and to avoid the drying of the samples during the measurement. The temperature of the samples was first stabilized from 4 to $25^{\circ} \mathrm{C}$ in $3 \mathrm{~min}$ with a controlled temperature ramp and kept at $25^{\circ} \mathrm{C}$ for $5 \mathrm{~min}$. Finally, the $G^{\prime}$ value was measured at $25^{\circ} \mathrm{C}$, which is the typical temperature in an irradiation room. In the case of liquid PAGAT, the temperature ramp went from 35 to $25{ }^{\circ} \mathrm{C}$ in $3 \mathrm{~min}$ and kept at that temperature for $5 \mathrm{~min}$. Every sample was measured at a constant strain of $5 \%$ and frequency range from 0.1 to $100 \mathrm{~Hz}$. Three different samples of the same material were analyzed for statistical purposes. In order to quantify the effect of adding the inorganic salts or modifying the gelatin matrix on the rheological properties of the materials, elastic modulus variation $(E M V)$ was defined as the ratio between the elastic modulus $\left(G^{\prime}\right)$ of the modified sample and of the reference sample, as expressed in Equation (4).

$E M V=\frac{G_{T e s t}^{\prime}-G_{R e f}^{\prime}}{G_{\text {Ref }}^{\prime}}$

Where $G^{\prime}{ }_{\text {Test }}$ and $G_{\text {Ref }}^{\prime}$ are the elastic moduli of the test and reference sample, respectively.

The expanded uncertainties in the mean values of $G^{\prime}$ were obtained by using Equation (3), where $U$ represents the uncertainty in the mean value of $G^{\prime}$ between 0.1 and $1 \mathrm{~Hz}, k$ represents a coverage factor for a Student's t-distribution with $67 \%$ of confidence, $x_{i}$ represents the mean $G^{\prime}$ value for each frequency and $u_{x_{i}}$ is the standard deviation of each $G^{\prime}$ mean value.

\subsection{Optical transmission}

In order to study the spatial dose distribution in the dosimeters, layer type containers were used in a 2D irradiation setup with PAGAT, PAGAT doped with $\mathrm{MgCl}_{2}$ and PAGAT doped with $\mathrm{MgCl}_{2}$ with $0.08 \% \mathrm{w} / \mathrm{v}$ of GTA. The dosimeters were analyzed by optical transmission with an apparatus described elsewhere (Vedelago et al., 2016). Optical transmission images of the dosimeters were acquired with a $580 \mathrm{~nm}$ filter before their irradiation and at different times until $48 h$ after irradiation, while controlling the temperature at $24 \pm 1{ }^{\circ} \mathrm{C}$. From the acquired images, optical density difference $(\triangle O D)$ was calculated by means of Equation (5).

$\Delta O D_{i, j}(t)=\frac{1}{d} \log \left[\frac{T_{N o-I r r}(i, j, t)}{T_{I r r}(i, j, t)}\right]$

Where $T_{I r r}(i, j, t)$ and $T_{N o-I r r}(i, j, t)$ are the transmitted light intensity maps for the irradiated and non irradiated samples at different times for each $(i, j)$ pixel, and $d$ is sample width. A region of interest (ROI) of $75 \times 75$ pixels $\left(2.163 \times 2.163 \mathrm{~cm}^{2}\right)$ that excludes the edges of the dosimeter was used for each $\triangle O D$ map. Then, a temporal distribution variation map (TDV) was generated by means of Equation (6)

$T D V(i, j, t)=\frac{\Delta O D_{R O I}(i, j, t)}{\Delta O D_{R O I}(i, j, 0)}$

From the obtained TDVs, changes in the post-irradiation response of the dosimeters can be evaluated over time. In order to quantify these changes, histograms were generated for each $T D V$ and fitted with a lognormal distribution function. The mean value of the lognormal fitted functions is strictly related to the temporal stability of the dosimetric system.

Also, the capability of each material to preserve the spatial dose distribution was analyzed by a thresholding method where the minimum $\triangle O D$ value of the irradiated zone of $\triangle O D_{R O I}(i, j, 0)$ was used as threshold value. This method resulted in binary maps $I(i, j, t)$ from where the spatial distribution variation $\operatorname{SDV}(i, j, t)$ was calculated by means of Equation (7). Finally, changes in the $S D V$ were quantified by evaluating the proportion of $\operatorname{SDV}(i, j, t)$ that differ from the ones of samples just after their irradiation, as expressed in Equation (8).

$S D V(i, j, t)=|I(i, j, t)-I(i, j, 0)|$

$\Delta S D=\frac{\text { Number of pixels with value }=1}{\text { Number of total pixels }} \times 100$

\section{Results}

\subsection{Effect of crosslinking of the gelatin matrix on the dosimetric system}

Fig. 2 contains the change in the elastic modulus $\left(G^{\prime}\right)$ of the dosimetric material due to GTA crosslinking of the gelatin matrix. In Fig. 1a, the rheometry results for the samples PAGAT (P1) and PAGAT with $0.26 \% w / v, 0.15 \% w / v$ and $0.08 \% w / v$ are depicted. Fig. $2 \mathrm{~b}$ presents the mean values of $\mathrm{G}^{\prime}$ for frequencies between 0.1 and $1 \mathrm{~Hz}$.

The results of dose response of dosimeters with and without crosslinking are presented in Fig. 3, pointing out that higher GTA crosslinking leads to lower sensitivities (Fig. 3b).

\subsection{Effect of inorganic salts on the dosimetric system}

The effect of adding the inorganic salts to the dosimetric system on the rigidity of the gelatin matrix is depicted in Fig. 4. Mean $G^{\prime}$ values for 


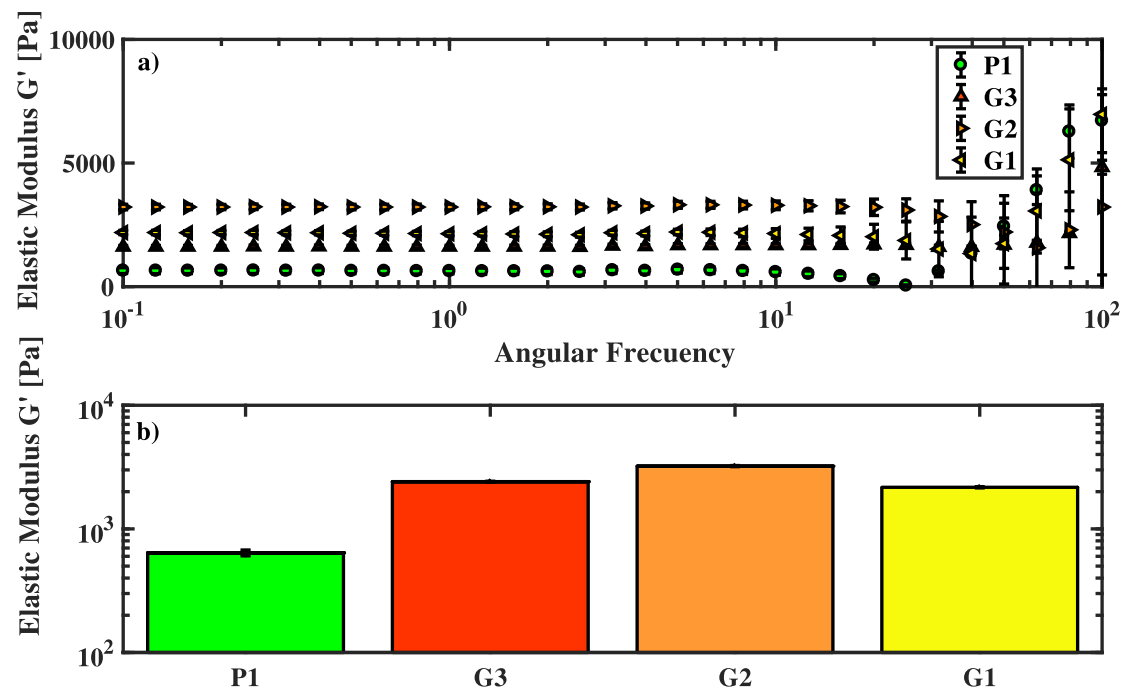

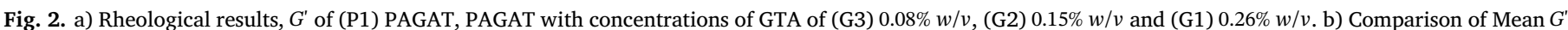

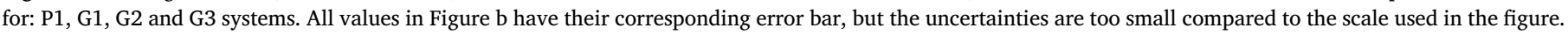

materials P2, P3, and P4 containing $\mathrm{MgCl}_{2}, \mathrm{CaCl}_{2}$ and $\mathrm{MnCl}_{2}$ are presented. Also, values of PAGAT (P1) and a liquid state PAGAT (P5) are included. All materials, except P1, exhibited very low $G^{\prime}$ values, which are not in the range of typical viscoelastic materials.

Dose response of these materials are summarized in Fig. 5. There was a significant increase in the dose sensitivity in materials with $\mathrm{MgCl}_{2}$ (P2), $\mathrm{CaCl}_{2}$ (P3) and liquid state PAGAT (P5) compared to PAGAT (P1). On the other hand, $\mathrm{MnCl}_{2}$ resulted in a non responsive material to $\mathrm{X}$-ray radiation, at least within the dose range of the present study.

\subsection{Effect of crosslinking on a dosimetric system containing $\mathrm{MgCl}_{2}$}

A complete stability study was carried out with the dosimetric system with the highest dose sensitivity, namely PAGAT with $\mathrm{MgCl}_{2}$. The effect of crosslinking the gelatin matrix with GTA with concentrations of $0.26 \% w / v, 0.15 \% w / v$ and $0.08 \% w / v$ in this material is presented in Fig. 6. A clear increase of the $G^{\prime}$ for all the materials was observed with higher concentrations of GTA, obtaining similar or higher rigidities to the one of PAGAT.

a)
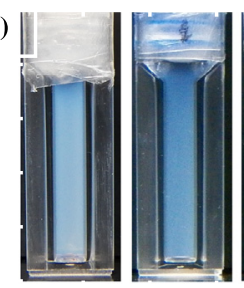

P1

G3

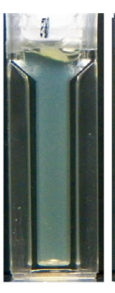

G2

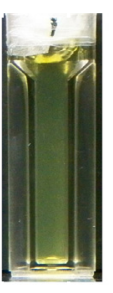

G1

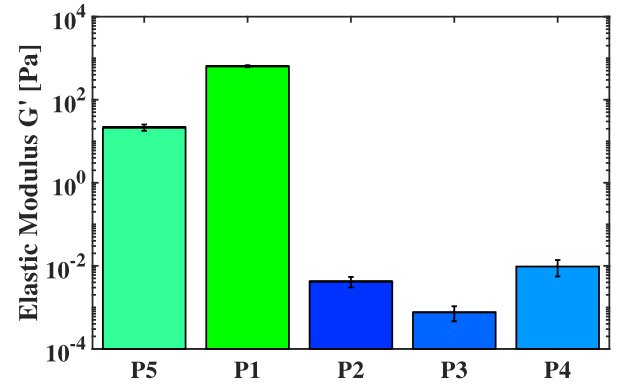

Fig. 4. Mean $G^{\prime}$ values for (P1) PAGAT, (P5) liquid PAGAT and PAGAT with (P2) $\mathrm{MgCl}_{2}$, (P3) $\mathrm{CaCl}_{2}$ and (P4) $\mathrm{MnCl}_{2}$.

Fig. 7 reports the corresponding dose response for these materials. Only PG3 presented a dose sensitivity higher than the one of PAGAT with a $75 \%$ increase in the sensitivity characterized by the fitted slope.

Table 2 summarizes the rheological and dose sensitivity results for all studied dosimetric systems. These results indicate that a relationship

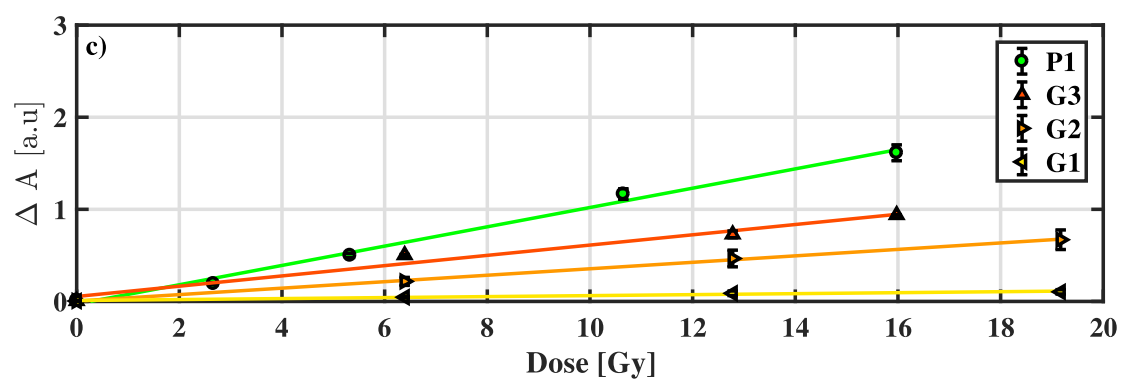

Fig. 3. Crosslinking effect on the dose response. a) Dosimetric systems uniformly irradiated with the same dose. b) Effect of GTA concentration on the sensitivity of PAGAT. c) Dose response curves of the different systems. Reported error bars represent the expanded uncertainties with $67 \%$ of confidence. 
a)

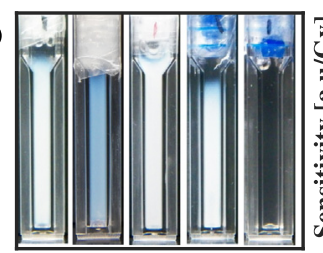

$\begin{array}{lllll}\text { P5 } & \text { P1 } & \text { P2 } & \text { P3 } & \text { P4 }\end{array}$
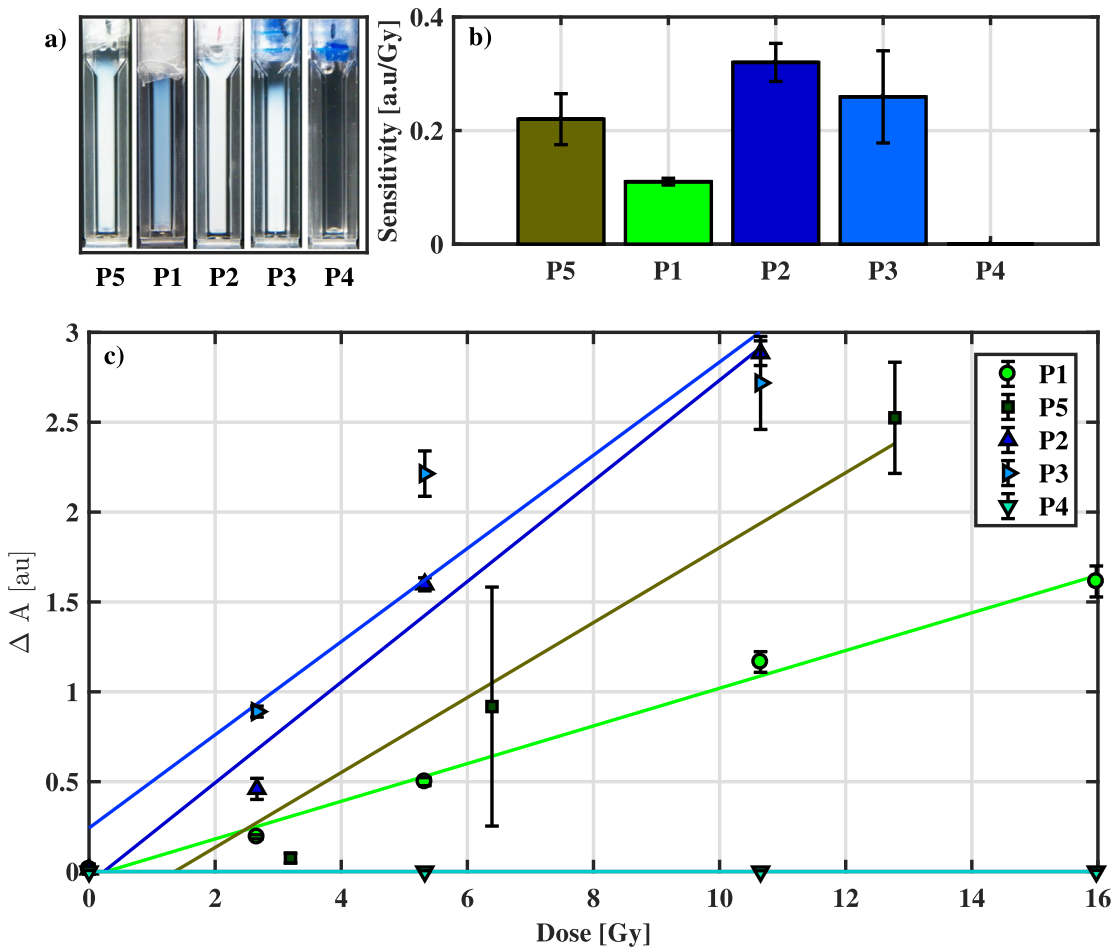

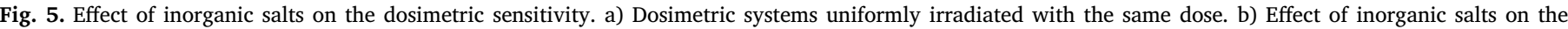

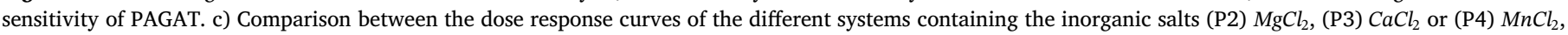
(P1) PAGAT and (P5) liquid PAGAT. Reported error bars represent the expanded uncertainties with $67 \%$ of confidence.

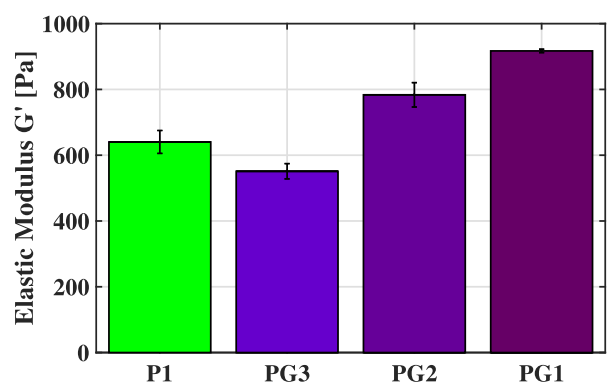

Fig. 6. Mean $G^{\prime}$ values for (P1) PAGAT and PAGAT with $\mathrm{MgCl}_{2}$ with GTA concentrations of (PG1) $0.26 \% w / v$, (PG2) $0.15 \% w / v$ and (PG3) $0.08 \% w / v$. Reported error bars represent the expanded uncertainties with $67 \%$ of confidence.

between the elastic modulus $G^{\prime}$ and the sensitivity of the material could exist.

3.3.1. Effect of GTA crosslinking on temporal and spatial stability of PAGAT dosimeters with $\mathrm{MgCl}_{2}$

Fig. 8 shows the results of the optical density for three different post irradiation times in the layer type dosimeters PAGAT (P1), PAGAT with $\mathrm{MgCl}_{2}$ (P2) and PAGAT with $\mathrm{MgCl}_{2}$ crosslinked with GTA $(0.08 \% \mathrm{w} / \mathrm{v})$ (PG3). Spatial dose distribution stability of systems P1 and PG3 appears to be adequate during the first $24 h$, but P2 system exhibits non negligible degradation $3 h$ after its irradiation.

From these $\triangle O D$ results, TDV histograms were generated and temporal stability information was determined as described in Appendix A. The mean value of lognormal distributions fitted to the TDV histograms are represented in Fig. 9 at different post irradiation times with their respective standard deviations. Results for $\mathrm{P} 1$ indicate that a maximum response is achieved after $5 h$ and remains stable for at least $48 h$. The response of PG3 increases continuosly during the whole $48 h$ interval. Finally, P2 does not reach any stable value and the signal completely disappears after $10 \mathrm{~h}$.

Fig. 10 reports the $\Delta S D$ results, which provide information on the spatial stability of the dosimeters and therefore on the diffusion or displacements of the irradiated material within the dosimeter. From the figure it becomes clear that both P1 and PG3 are very stable regarding to spatial stability and that P2 have an unstable behavior even at very short times after its irradiation. It is worthwhile mentioning that the maximum possible change in $\triangle S D$ is the percentage of pixels of the image $\triangle O D_{R O I}(i, j, 0)$ with an $\triangle O D$ above the threshold value. Thus, there was no spatial distribution stability in P2, which had around of $40 \%$ of its initial pixels above the threshold value, with a post irradiation time of $5 \mathrm{~h}$.

\section{Discussion}

\subsection{Gelatin matrix crosslinking}

As expected, an increase in the GTA concentration during the crosslinking reaction of the gelatin leads to materials with higher rigidity and elastic modulus. A different trend was only observed for the reactions carried out with a GTA concentration of $0.26 \% \mathrm{w} / \mathrm{v}$, where a decrease in the $G^{\prime}$ value was observed when compared with the one obtained with $0.15 \% w / v$ of GTA. However, this result was not unexpected and has been reported in a previous study (Romero et al., 2016). From a quantitative point of view, the elastic modulus variation (EMV) due to the addition of GTA were 2.40, 4.03 and 2.77 for systems with GTA concentrations of $0.26 \% w / v$ (G1), $0.15 \% w / v$ (G2) and $0.08 \% w / v$ (G3), respectively.

GTA effect on the dose response of PAGAT was significative as can be seen in Fig. 3, where SV values of $-0.95,-0.67$ and -0.47 were obtained for G1, G2, and G3 respectively. On the other hand, if the rigidity of these materials are considered in the analysis a relationship between $G^{\prime}$ and dose sensitivity could be inferred. A higher crosslinking in the structure of the dosimeters leads to more complex materials where the mobility of the reactive species become hindered or affected 
a)

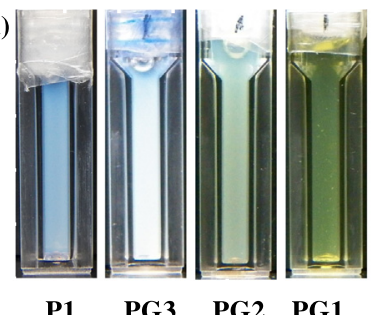

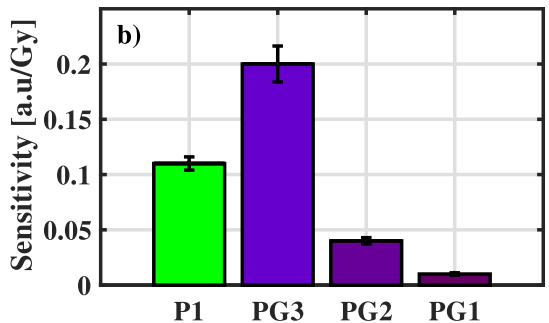

P1 PG3 PG2 PG1

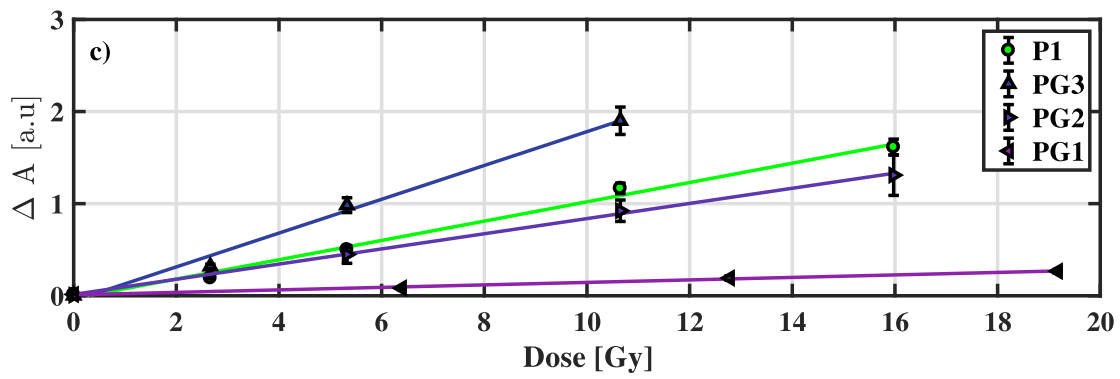

Fig. 7. Comparison between PAGAT and PAGAT with $\mathrm{MgCl}_{2}$ in terms of the effect of GTA concentration on the sensitivity. Reported error bars represent the expanded uncertainties with $67 \%$ of confidence.

Table 2

Radiosensitive material properties (dose response sensitivity and elastic modulus).

\begin{tabular}{lllll}
\hline Set & Sensitivity $\left[\mathrm{Gy}^{-1}\right]$ & SV & G' $[\mathrm{Pa}]$ & EMV \\
\hline P1 & $(1.05 \pm 0.06) \times 10^{-1}$ & 0.00 & $(6.40 \pm 0.35) \times 10^{2}$ & 0.00 \\
P2 & $(2.80 \pm 0.34) \times 10^{-1}$ & 1.67 & $(4.21 \pm 1.2) \times 10^{-3}$ & -1.00 \\
P3 & $(2.59 \pm-0.81) \times 10^{-1}$ & 1.47 & $(7.60 \pm 3.00) \times 10^{-4}$ & -1.00 \\
P4 & $(-4.79 \pm 1.00) \times 10^{-4}$ & -1.00 & $(9.63 \pm 4.10) \times 10^{-3}$ & -1.00 \\
P5 & $(2.08 \pm 0.45) \times 10^{-1}$ & 0.99 & $(2.16 \pm 0.37) \times 10^{1}$ & -0.97 \\
G1 & $(5.33 \pm 0.90) \times 10^{-3}$ & -0.95 & $(2.17 \pm 0.03) \times 10^{3}$ & 2.40 \\
G2 & $(3.51 \pm 0.12) \times 10^{-2}$ & -0.67 & $(3.22 \pm 0.34) \times 10^{3}$ & 4.03 \\
G3 & $(5.58 \pm 0.82) \times 10^{-2}$ & -0.47 & $(2.41 \pm 0.02) \times 10^{3}$ & 2.77 \\
PG1 & $(1.35 \pm 0.11) \times 10^{-2}$ & -0.87 & $(9.17 \pm 0.05) \times 10^{2}$ & 0.43 \\
PG2 & $(8.23 \pm 0.29) \times 10^{-2}$ & -0.21 & $(7.84 \pm 0.37) \times 10^{2}$ & 0.22 \\
PG3 & $(1.84 \pm 0.16) \times 10^{-1}$ & 0.75 & $(5.51 \pm 0.23) \times 10^{2}$ & -0.14 \\
\hline
\end{tabular}

because of the formation of more rigid pores in the gelatin matrix containing enclosed water molecules. What is more, the chemical reaction between GTA and the protein molecules of gelatin involves the amino groups in the protein, which are implicated in the hydration and mobility of the reactants as well, thus reducing the available water molecules in the dosimeter leading to a lower reactivity. When GTA is added in excess a competitive reaction between GTA and amino groups in the gelatin and between GTA with another GTA molecule takes place. This competitive reaction produces gelatin structures functionalized with free aldehyde chemical groups, which are typically found in their hydrated form (Wolfel et al., 2017), thus reducing water availability and mobility of reactant species in the dosimeters, in spite of the decrease in the elastic modulus. Similar results were obtained in studies of gelatin concentration on AAm and BIS systems (Lepage et al., 2001), where an increase in the rigidity of the structure of the dosimeter due to an increase in gelatin concentration led to a lower sensitivity and higher saturation dose. Finally, a higher crosslinking in the dosimeters and its
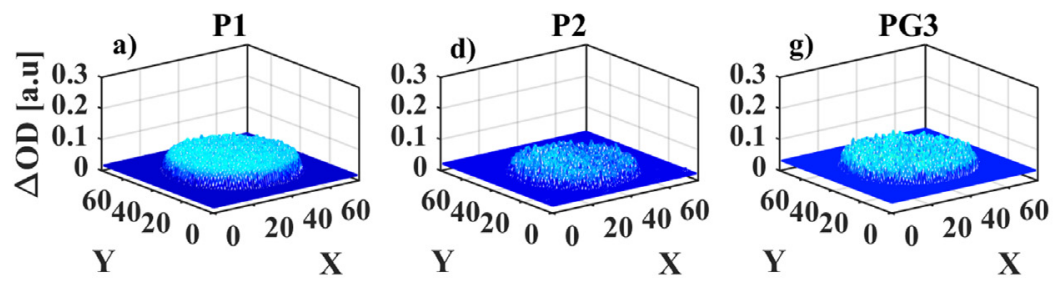

oh
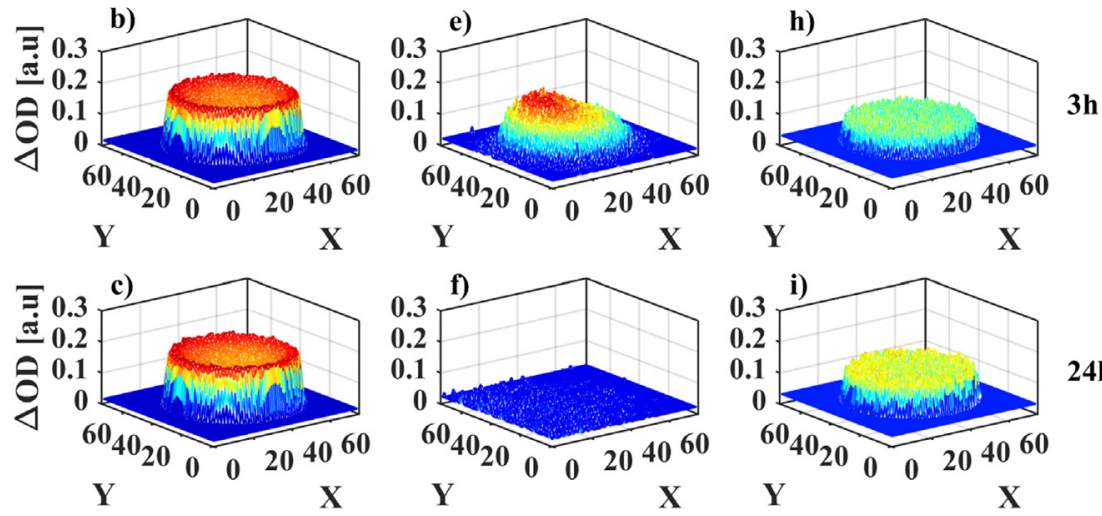

24h

Fig. 8. Optical density differences at different post irradiation times ( $0 h, 3 h$ and $24 h$ ) for P1 (a, b y c), P2 (d, e y f) and PG3 (g, h y i) systems. 

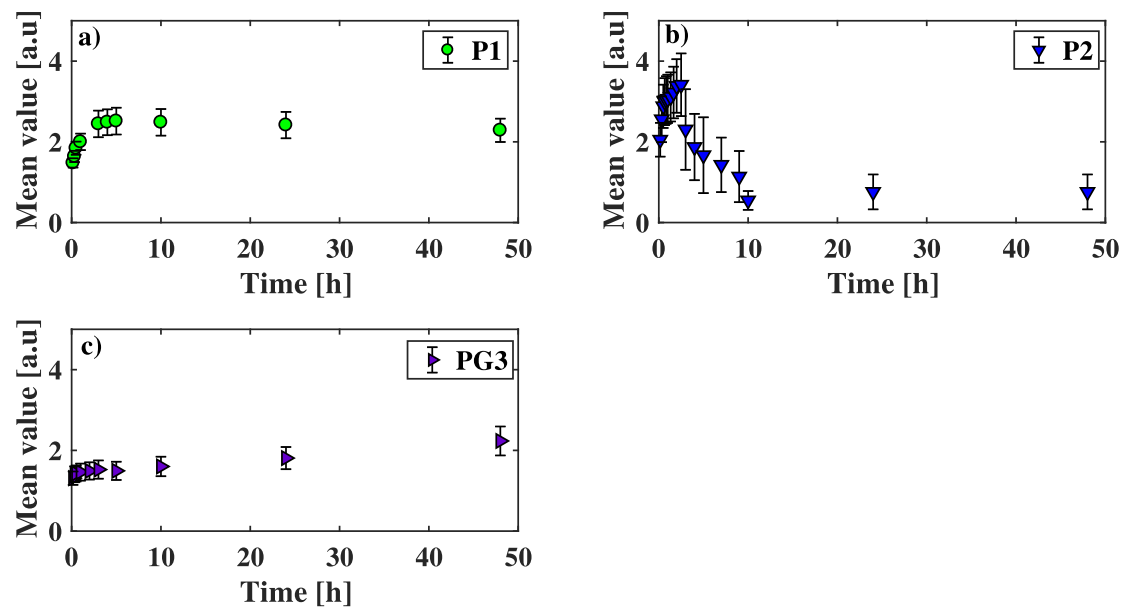

Fig. 9. Temporal stability behavior interpreted from the mean value in the lognormal TDV distribution for P1, P2 and PG3.
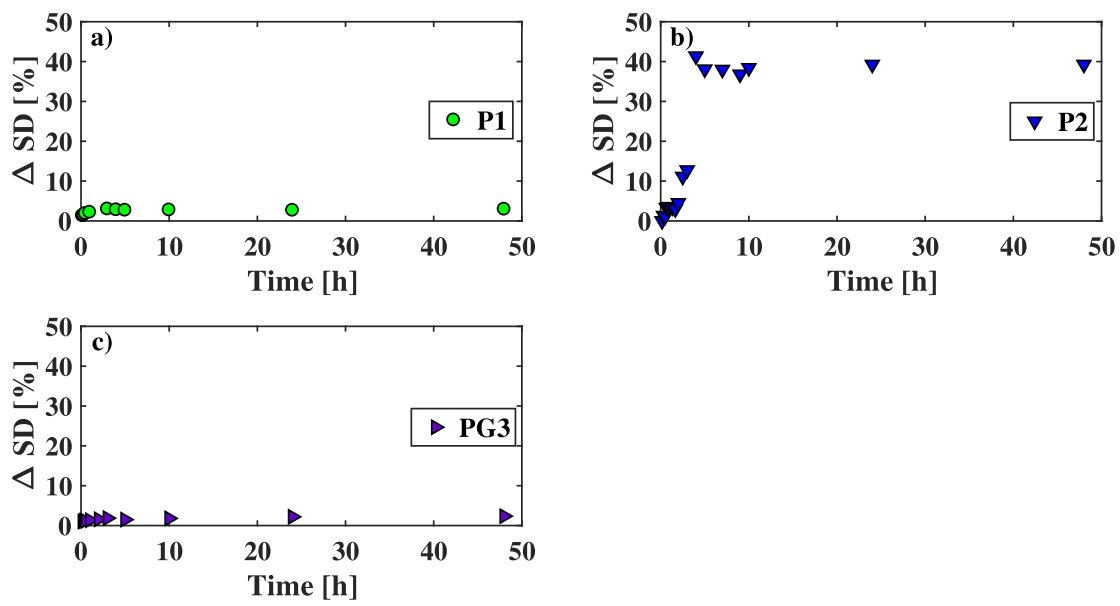

Fig. 10. Spatial distribution change $(\Delta S D)$ for $\mathrm{P} 1$, P2 and PG3.

consequent higher elastic modulus didn't produce any offset in the non irradiated dosimeters as reported in studies with higher gelatin concentrations (De Deene et al., 2006).

\subsection{Elastic modulus and dosimetric sensitivity analysis}

The information of the $G^{\prime}$ value of liquid PAGAT was extremely useful to analyze the results of materials with inorganic salts. From the results presented in Fig. 4, comparable $G^{\prime}$ reductions were obtained for liquid PAGAT and systems with inorganic salts. All these materials had $G^{\prime}$ values $\leq 10^{1} P a$, which are not typical for viscoelastic materials. On the other hand, materials doped with $\mathrm{MgCl}_{2}$ and $\mathrm{CaCl}_{2}$ presented enhanced performance regarding to their dosimetric sensitivity (Fig. 5) with SV values of 1.67 and 1.47 respectively. Liquid state PAGAT also presented a SV enhancement of 0.99 . If the rheometry and sensitivity results are analyzed together, it becomes unclear whether the increase in the dose sensitivity is because of the presence of salt ions or due to the liquefaction of the gelatin matrix. Indeed, it is more likely a combination of both effects, where most of the sensitivity improvement is related to the melting point depression of the gelatin matrix as suggested by the obtained results. These materials have serious limitations as $3 \mathrm{D}$ dosimeters because of the loss in their spatial and temporal stability. Finally, the polymerization within the PGD was completely inhibited when $\mathrm{MnCl}_{2}$ was incorporated, probably because $\mathrm{Mn}^{+2}$ ions are able to form complexes with other molecules than water (Hikichi et al., 1990), thus restricting not only water availability but also limiting the mobility of monomer species and consequently hindering the polymerization reaction in the dosimeters.

\subsection{Dose response of dosimeters with $\mathrm{MgCl}_{2}$ crosslinked with GTA}

PAGAT with $\mathrm{MgCl}_{2}$ was stabilized with different GTA concentrations obtaining materials with rigidity similar or above the one of PAGAT. However, the dose response of the dosimeters was reduced when higher concentrations of GTA were used. In this case, two opposite effects were present. On one hand, the $\mathrm{Mg}^{+2}$ ions have an enhancement effect on the polymerization reaction that would increase the sensitivity of the material as Hayashi et al. reported (Hayashi et al., 2013), but on the other hand the use of $\mathrm{Mg}^{+2}$ requires the crosslinking of the gelatin matrix to keep its spatial distribution, which entails a loss in the sensitivity of the dosimeter. Therefore, by properly selecting the GTA $/ \mathrm{Mg}^{+2}$ concentrations it should be possible to obtain a material with better dose response and similar mechanical properties than PAGAT. With that in mind, the systems P1, PG1, PG2 and PG3 were compared obtaining EMV values of $0.00,0.43,0.22$ and -0.14 respectively, and SV values of $0.00,-0.87$, -0.21 and 0.75 , respectively. PG3 was the only material with enhanced sensitivity compared to PAGAT and still able to maintain spatial information within time in this study.

\subsection{Stability análisis}

2D analysis of P1, P2 and PG3 provided information on the spatial and temporal stability of these materials. On the three systems an initial stabilization period was observed, where the polymerization initiated 
during the irradiation was still going on. The dose response of P1 and PG3 reached stable values after $6 \mathrm{~h}$ from their irradiation, as indicated by a constant $T D V$ value. In contrast, P2 became unstable with only $3 \mathrm{~h}$ due to the high diffusion present in this material, leading to a complete loss of dose distribution within $10 \mathrm{~h}$ from its irradiation. The use of GTA as a crosslinking agent was very efficient but increased the optical absorbance of the materials within time, leading to a slight yellow coloration after $24 \mathrm{~h}$. Nevertheless, this effect was not a limiting factor in the stability analysis and only caused a slight increase in the $\triangle O D$ values of stable dosimeters at long post irradiation times. Spatial stability of these dosimeters was also studied, P1 and PG3 presented remarkable spatial stability with values of $\Delta S D$ lower than 3\%. On the contrary, for non stabilized systems like P2 a complete loss of the spatial information was observed and the highest possible $\Delta S D$ value was reached with just $5 \mathrm{~h}$ from its irradiation.

\section{Conclusions}

The use of inorganic charges to improve the dosimetric response of polymer gel dosimeters entails a loss in the stability of their structure and, as a consequence, a poor performance to register 3D dose distributions. In this study $\mathrm{Mg}^{+2}, \mathrm{Mn}^{+2}$ and $\mathrm{Ca}^{+2}$ were used in PAGAT dosimeters obtaining in all cases liquid-like materials exhibiting higher $\mathrm{X}$-ray sensitivities but a complete loss of the spatial dose distribution within $5 \mathrm{~h}$ from their irradiation. To overcome these limitations a chemical crosslinking reaction with glutaraldehyde was used and the mechanical properties of the dosimeters were improved achieving similar elastic moduli to the one of PAGAT. Moreover, thanks to the crosslinking of the gelatin matrix, the stability of $2 \mathrm{D}$ dose distributions was evaluated comparing PAGAT, PAGAT doped with $\mathrm{MgCl}_{2}$ and crosslinked PAGAT doped with $\mathrm{MgCl}_{2}$. The temporal evolution of the Xray response along with spatial distribution variations, mainly because of diffusion or displacement of the polymerized regions of the dosimeters, were also quantified. In every case an initial stabilization period of the response was observed of about $10 \mathrm{~h}$ with no significant differences because of the crosslinking of the gelatin matrix. The spatial distribution and stability of both PAGAT and crosslinked PAGAT with $\mathrm{MgCl}_{2}$ dosimeters did not differ significatively between each other, while being ten times lower than the one of PAGAT doped with $\mathrm{MgCl}_{2}$. Correlation between the rigidity of the gelatin matrix and the sensitivity of the dosimeters was observed, probably due to the availability of water molecules that becomes affected by reducing the water spaces and by reducing the number of amine groups in the gelatin matrix. What is more, if an excess of crosslinker is used in the reaction, the sensitivity in the dosimeters becomes even lower due to the hydration of terminal aldehyde free groups formed in the gelatin matrix and its consequent reduction of available water molecules for the monomers involved in the polymerization reactions. Among the studied ions, $\mathrm{Mg}^{+2}$ and $\mathrm{Ca}^{+2}$ exhibited similar effects over the dosimetric performance of the materials, being the former the one with highest sensitivity. On the other hand, the response of dosimeters containing $\mathrm{Mn}^{+2}$ was completely suppressed, in spite of having a similar crystal ionic radius and effect over the structure of water to the other studied ions. Finally, a dosimeter with a sensitivity enhancement of $75 \%$ and similar mechanical properties compared to PAGAT was obtained by properly selecting the concentration of glutaraldehyde in the crosslinking of the gelatin matrix and using $\mathrm{MgCl}_{2}$.

\section{Acknowledgments}

This study was partially financed by CONICET by means of the Project ESPORA IePIP 112-20130100658CO, PIP 112-0.20110101029 and SeCyT-UNC by means of DOSCOM I project and INSPIRATE I program. This project was also partially supported by Universidad de La Frontera by DIUFRO DI6008-16 and DI1003-17 projects. The authors would also like to thank to Universidad Nacional (UNA), Heredia, Costa Rica for the financial support (JB-C 0612-2014) of the PhD studies of D. Chacón.

\section{Appendix A. Temporal and spatial stability analysis in layer dosimeters}

The following methodology was used to analyze the temporal and spatial stability in 2D layer type dosimeters.

1. Optical transmission maps ( $T$ ) were obtained from the layer dosimeters prior to their irradiation $\left(\operatorname{Tr}_{0}\right)$ and at different times from 0 to $48 h$ after their irradiation. Typical optical transmission maps are depicted in Figure A1.

2. Optical density difference $(\triangle O D)$ was calculated by means of Equation (A.1) for each transmission map and $\triangle O D$ maps were obtained. Typical $\triangle O D$ maps are shown in Figure A2.

$\Delta O D_{i, j}(t)=\frac{1}{d} \log \left(\frac{T r_{i, j}(0)}{\left.T r_{i, j} t\right)}\right)$

3. A region of interest $(\mathrm{ROI})$ of $75 \times 75$ pixels centered in the irradiated area was defined for the subsequent analyses and calculations, in order to discard the outer boundary regions of the $\triangle O D$ map.

4. A temporal distribution variation parameter (TDV) was defined according to Equation (A.2) by using the $\triangle O D$ information in the selected ROI of the $\triangle O D$ map at $0 h$ after the irradiation as a reference state. Typical TDV maps are shown in Figure A3.

$T D V(i, j, t)=\frac{O D_{R O I}(i, j, t)}{O D_{R O I}(i . j .0)}$

5. Histograms were generated from the TDV maps by using home made routines programmed in MATLAB (version 7.11.0.584 R2010b 64 bit) software (MathWorks Inc, Natick, MA, USA). TDV values with no changes at all, identified in this method as TDV values between 0.99 and 1.01 , were discarded for the subsequent analyses in order to account only for the regions with changes in the TDV. Figure A4 depicts typical histograms for $T D V$ maps obtained in this study, which correspond to the TDV maps presented in Figure A3.

6. The $T D V(i, j, t)$ distribution histograms were fitted to a lognormal function with the expression presented in Equation (A.3). Typical results of the fitted functions together with the original histograms are presented in Figure A5

$f(x ; \mu, \sigma)=\frac{1}{\sqrt{2 \pi \sigma x}} e^{-\left[\ln (x)-\mu^{2}\right] /\left(2 \sigma^{2}\right)}$

7. To evaluate the temporal stability, the mean value and standard deviation of the lognormal distribution were calculated by means of Equation 
(A.4) and (A.5); respectively. Typical results of the temporal evolution of these parameters are presented in Figure A6.

mean value $=e^{\left(\mu+\frac{\sigma^{2}}{2}\right)}$

$s d=\sqrt{\sigma^{2}}=\operatorname{sqrt}\left(e^{\sigma^{2}}-1\right) e^{2 \mu+\sigma^{2}}$

8. Changes in the position of the irradiated zones due to diffusion or displacement were accounted as spatial distribution changes $(\triangle S D)$. For that purpose, a thresholding approach was used as expressed in Equation (A.6) in the selected ROI of the $\triangle O D$ maps, where $T$ represents the selected threshold value, defined as the lowest value in the irradiated region of the selected ROI of the $\triangle O D$ map at time $=0 h$. As a result, a binary map (I) was obtained. Typical I maps are depicted in Figure A7.

$I(i, j, t)=\left\{\begin{array}{lll}1 & \text { if } \quad \Delta O D(i, j, t) \geq T \\ 0 & \text { if } \quad \Delta O D(i, j, t)<T\end{array}\right.$

9. A spatial distribution variation parameter $(S D V)$ was calculated as expressed in Equation (A.7) and SDV maps were generated. Typical $S D V$ maps are presented on Figure A8.

$S D V(i, j, t)=|I(i, j, t)-I(i, j, 0)|$

10. Changes in the SDV $(\Delta S D)$ were calculated by means of Equation (A.8). Typical results at different post irradiation times are presented in Figure A9.

$\Delta S D=\frac{\text { Number of pixels with value }=1}{\text { Number of total pixels }} \times 100$
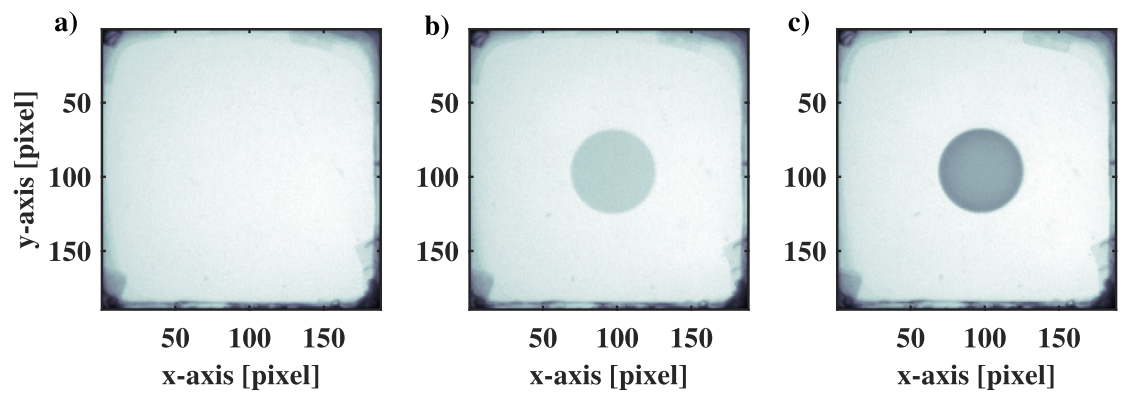

Fig. A1. Optical transmission maps of PAGAT layer dosimeters a) before irradiation, b) $0 h$ and c) $24 h$ after being irradiated.
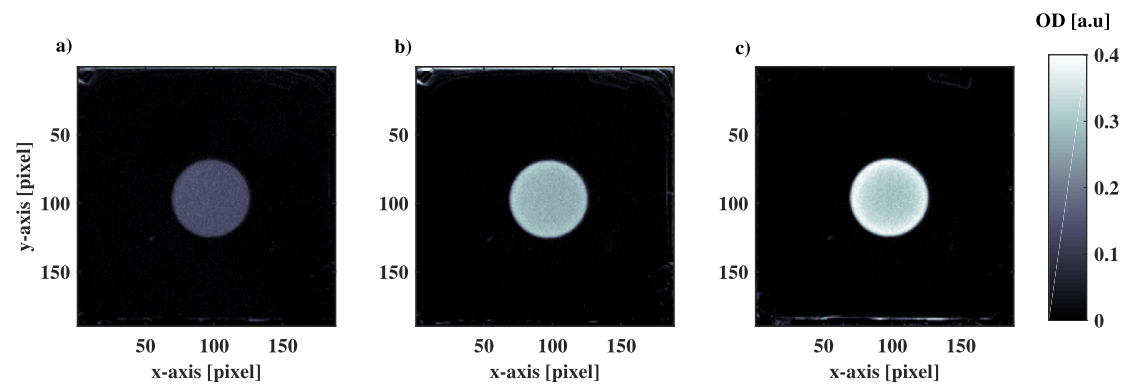

Fig. A2. $\triangle O D$ map of PAGAT at a) $0 h$, b) $10 \mathrm{~min}$ and c) $24 h$ from their irradiation.
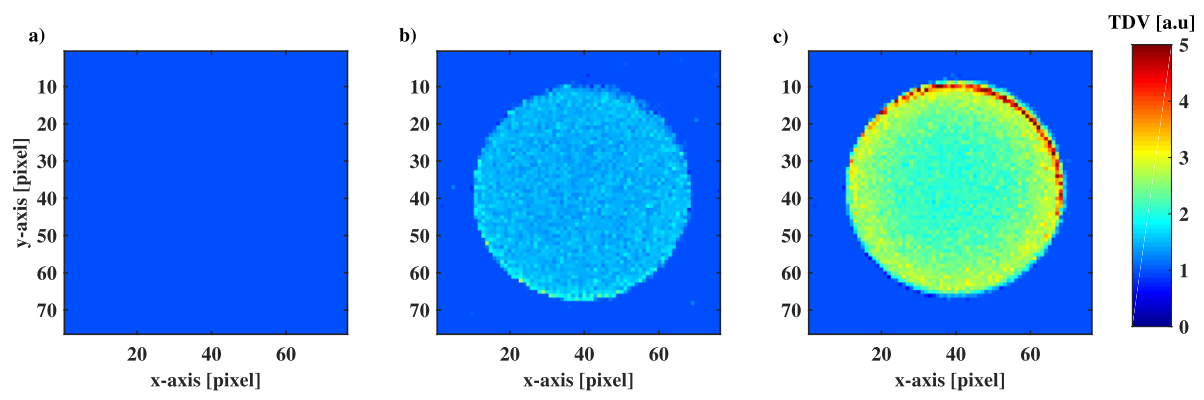

Fig. A3. TDV maps of PAGAT at a) $0 h$ b) $10 \mathrm{~min}$ and c) $24 \mathrm{~h}$ from their irradiation. 

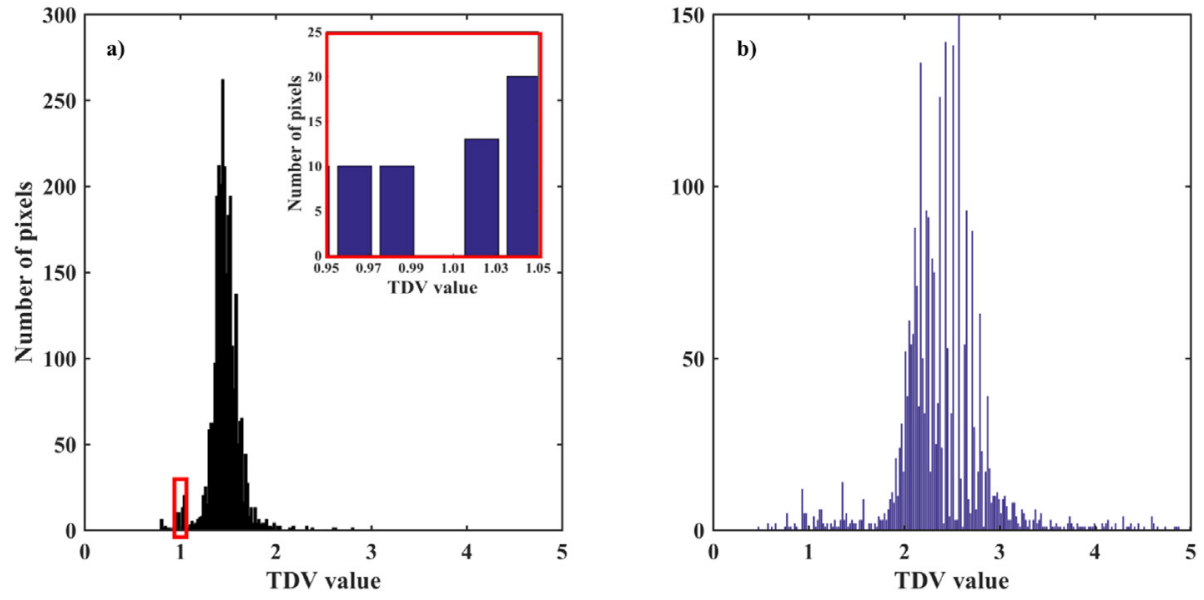

Fig. A4. TDV histograms for PAGAT at a) $10 \mathrm{~min}$ and b) $24 \mathrm{~h}$ from their irradiation.
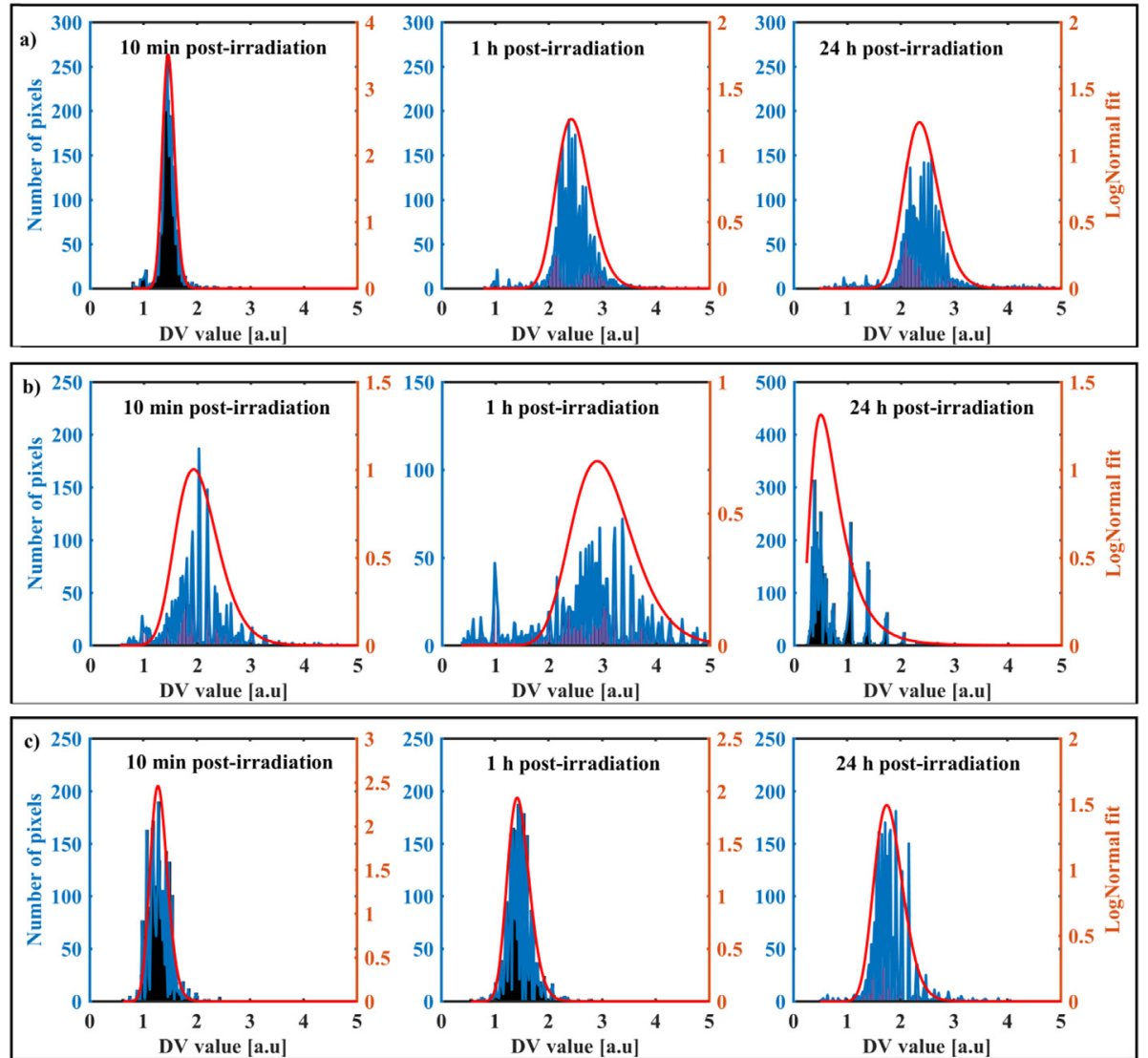

Fig. A5. TDV histograms and fitted lognormal distributions at different post irradiation times for a) PAGAT, b) PAGAT with $\mathrm{MgCl}_{2}$ and c) PAGAT with $\mathrm{MgCl}_{2}$ and $0.08 \% \mathrm{w} / \mathrm{v}$ of GTA.
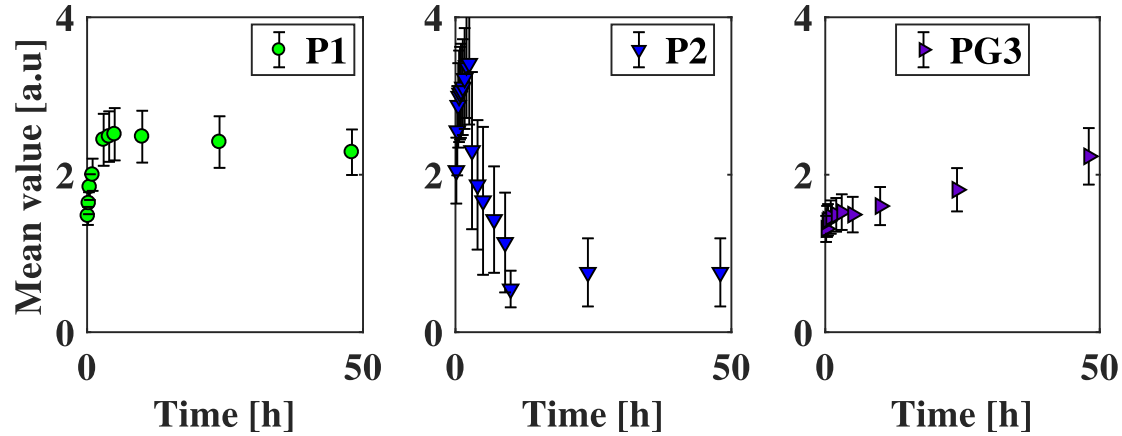

Fig. A6. Mean value vs post irradiation time of a) (P1) PAGAT, b) (P2) PAGAT with $\mathrm{MgCl}_{2}$ and c) (PG3) PAGAT with $\mathrm{MgCl}_{2}$ and $0.08 \%$ w/v of GTA. The standard deviation values are represented as error bars. 

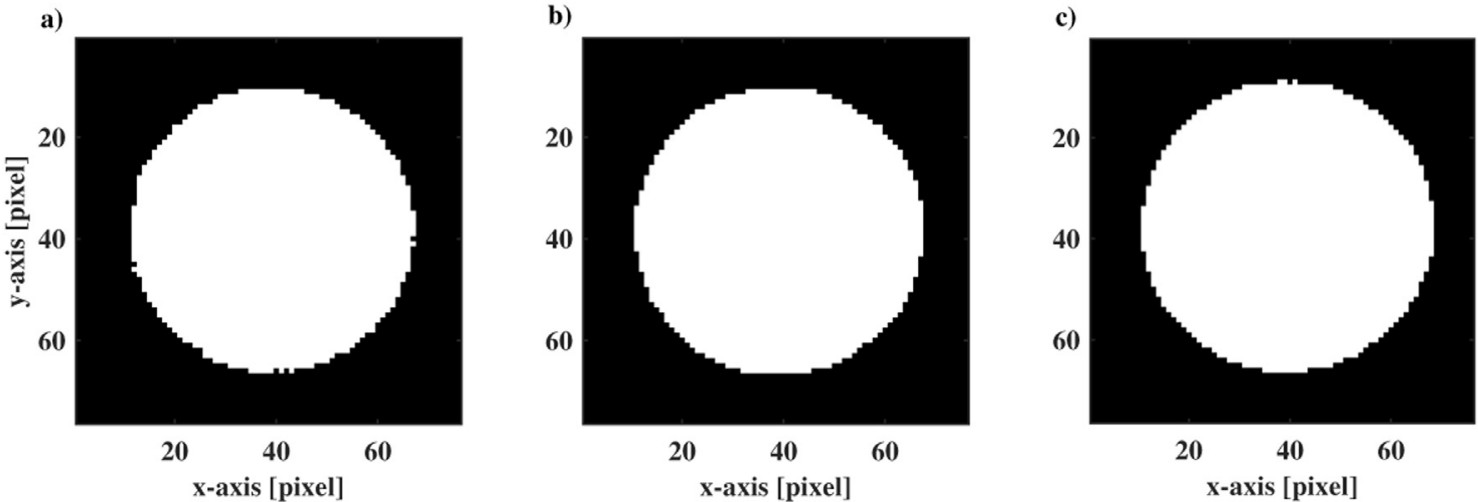

Fig. A7. Binary maps of PAGAT at a) $0 h$, b) $1 h$ and c) $24 h$ from their irradiation.

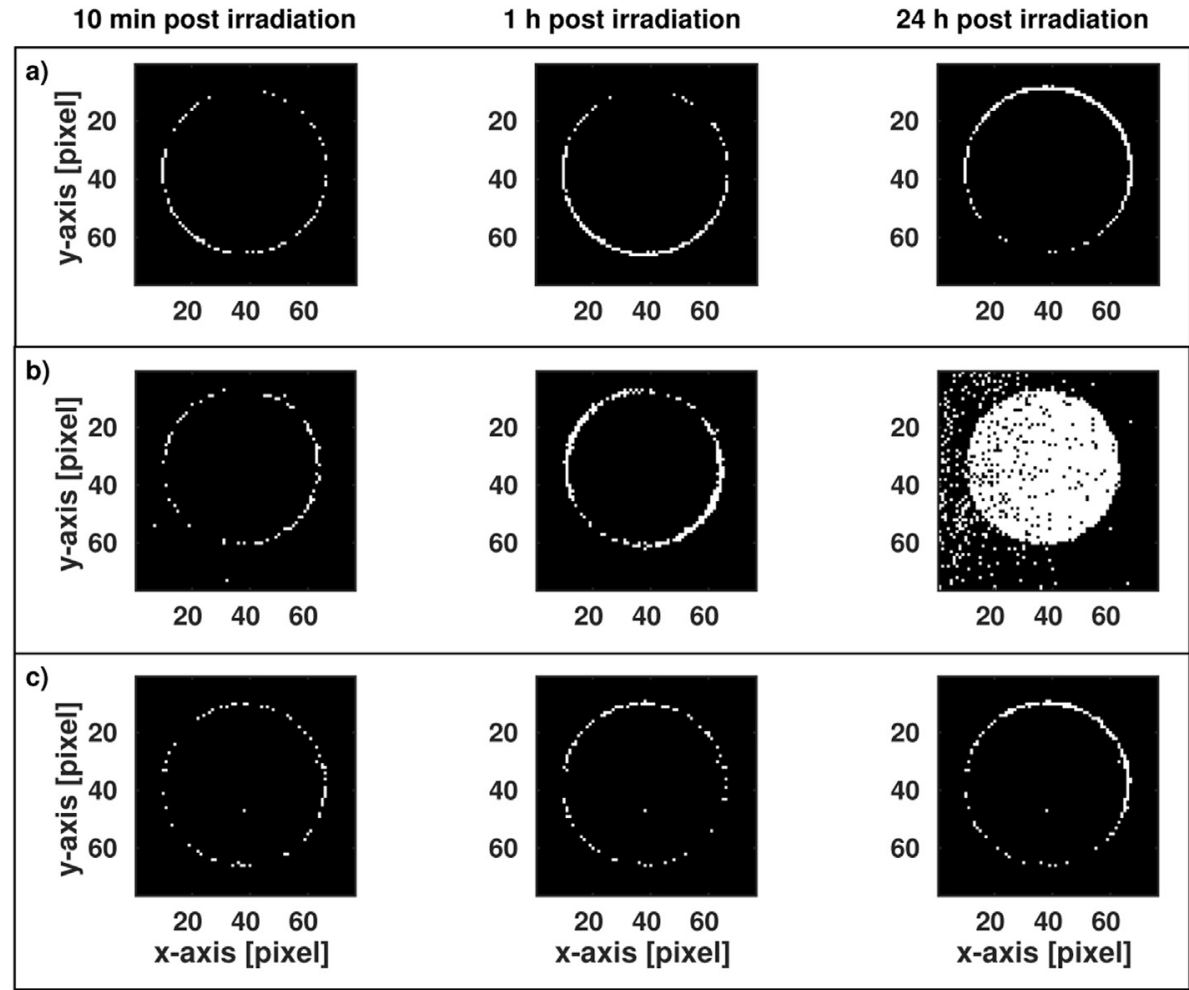

Fig. A8. SDV maps at different post irradiation times for a) (P1) PAGAT, b) (P2) PAGAT with $\mathrm{MgCl}_{2}$ and c) (PG3) PAGAT with $\mathrm{MgCl}_{2}$ and 0.08\% w/v of GTA.
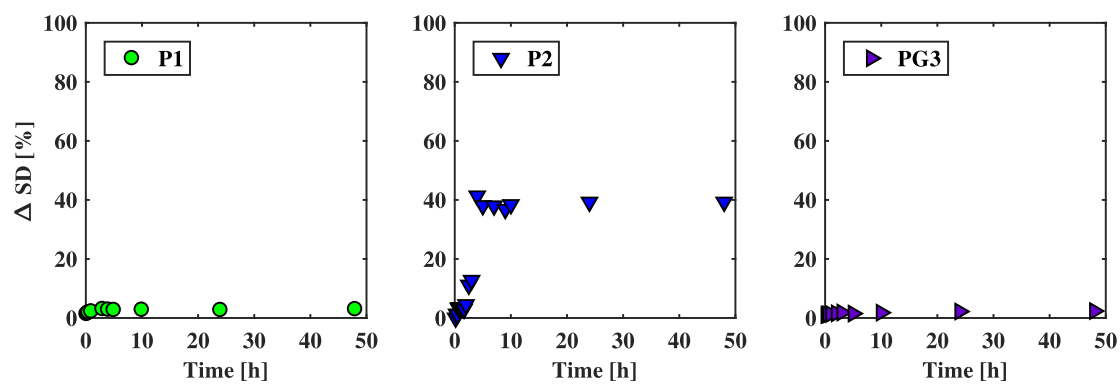

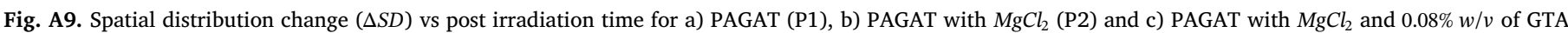
(PG3). 


\section{References}

Al-Jarrah, A., Rahman, A.A., Shahrim, I., Ab Razak, N.N.A.N., Ababneh, B., Tousi, E.T., 2016. Effect of inorganic salts and glucose additives on dose-response, melting point and mass density of genipin gel dosimeters. Phys. Med.: Eur. J. Med. Plants 32 (1), 36-41. https://doi.org/10.1016/j.ejmp.2015.09.003.

Aziz, S.A.A., Rahman, W.N.W.A., Abdullah, R., Harun, A.Z., Zakaria, A., 2013. Optimization of magat with formaldehyde gel dosimeter for dose evaluation. In: Proceeding of Global Engineering, Science and Technology Conference, ISBN 978-1922069-32-0.

Baldock, C., Deene, Y.D., Doran, S., Ibbott, G., Jirasek, A., Lepage, M., McAuley, K.B., Oldham, M., Schreiner, L.J., 2010. Polymer gel dosimetry. Phys. Med. Biol. 55 (5), R1. https://doi.org/10.1088/0031-9155/55/5/R01.

De Deene, Y., Vergote, K., Claeys, C., De Wagter, C., 2006. The fundamental radiation properties of normoxic polymer gel dosimeters: a comparison between a methacrylic acid based gel and acrylamide based gels. Phys. Med. Biol. 51 (3), 653. https://doi. org /10.1088/0031-9155/51/3/012.

Fernandes, J.P., Pastorello, B.F., de Araujo, D.B., Baffa, O., 2008. Formaldehyde increases MAGIC gel dosimeter melting point and sensitivity. Phys. Med. Biol. 53 (4), N53. https://doi.org/10.1088/0031-9155/53/4/N04.

Haug, I.J., Draget, K.I., Smidsrød, O., 2004. Physical and rheological properties of fish gelatin compared to mammalian gelatin. Food Hydrocolloids 18 (2), 203-213. https://doi.org/10.1016/S0268-005X(03)00065-1.

Hayashi, S.-i., Fujiwara, F., Usui, S., Tominaga, T., 2012. Effect of inorganic salt on the dose sensitivity of polymer gel dosimeter. Radiat. Phys. Chem. 81 (7), 884-888. https://doi.org/10.1016/j.radphyschem.2012.03.001.

Hayashi, S., Kawamura, H., Usui, S., Tominaga, T., 2013. Comparison of the influence of inorganic salts on the NMR dose sensitivity of polyacrylamide-based gel dosimeter. 444 (1), 012094. https://doi.org/10.1088/1742-6596/444/1/012094.

Hikichi, K., Tanaka, H., Konno, A., 1990. Nuclear magnetic resonance study of poly $(\gamma$ glutamic acid)—Cu (II) and Mn (II) complexes. Polym. J. 22 (2), 103. https://doi. org/10.1295/polymj.22.103.

Ibbott, G.S., 2004. Applications of gel dosimetry. J. Phys. Conf. 3 (1), 58-77. https://doi. org /10.1088/1742-6596/3/1/007.

Lepage, M., Whittaker, A.K., Rintoul, L., Bäck, S.Å.J., Baldock, C., 2001. The relationship between radiation-induced chemical processes and transverse relaxation times in polymer gel dosimeters. Phys. Med. Biol. 46 (4), 1061. https://doi.org/10.1088/ 0031-9155/46/4/311.

Marcus, Y., 2009. Effect of ions on the structure of water: structure making and breaking. Chem. Rev. 109 (3), 1346-1370. https://doi.org/10.1021/cr8003828.

Maryanski, M.J., Gore, J.C., Kennan, R.P., Schulz, R.J., 1993. NMR relaxation enhancement in gels polymerized and cross-linked by ionizing radiation: a new approach to
3D dosimetry by MRI. Magn. Reson. Imag. 11 (2), 253-258. https://doi.org/10. 1016/0730-725X(93)90030-H

Mattea, F., Chacón, D., Vedelago, J., Valente, M., Strumia, M.C., 2015. Polymer gel dosimeter based on itaconic acid. Appl. Radiat. Isot. 105, 98-104. https://doi.org/10. 1016/j.apradiso.2015.07.042.

Pavoni, J., Baffa, O., 2012. An evaluation of dosimetric characteristics of MAGIC gel modified by adding formaldehyde (MAGIC-f). Radiat. Meas. 47 (11-12), 1074-1082. https://doi.org/10.1016/j.radmeas.2012.10.004.

Rabaeh, K., Saion, E., Omer, M., Shahrim, I., Alrahman, A.A., Hussain, M., 2008. Enhancements in 3D dosimetry measurement using polymer gel and MRI. Radiat. Meas. 43 (8), 1377-1382. https://doi.org/10.1016/j.radmeas.2008.04.083.

Romero, M., Mattea, F., Vedelago, J., Chacón, D., Valente, M., Álvarez, C.I., Strumia, M., 2016. Analytical and rheological studies of modified gel dosimeters exposed to X-ray beams. Microchem. J. 127, 231-236. https://doi.org/10.1016/j.microc.2016.03.009.

Sarabia, A., Gómez-Guillén, M., Montero, P., 2000. The effect of added salts on the viscoelastic properties of fish skin gelatin. Food Chemistry 70 (1), 71-76. https://doi. org/10.1016/S0308-8146(00)00073-X.

Senden, R.J., De Jean, P., McAuley, K., Schreiner, L., 2006. Polymer gel dosimeters with reduced toxicity: a preliminary investigation of the NMR and optical dose-response using different monomers. Phys. Med. Biol. 51 (14), 3301. https://doi.org/10.1088/ 0031-9155/51/14/001.

Taylor, J., 1997. Introduction to Error Analysis, the Study of Uncertainties in Physica Measurements, second ed. Published by University Science Books, 648 Broadway, Suite 902, New York, NY 10012 ISBN 0-935702-42-3.

Titus, D., Samuel, E.J.J., Roopan, S.M., 2016. Current scenario of biomedical aspect of metal-based nanoparticles on gel dosimetry. Appl. Microbiol. Biotechnol. 100 (11), 4803-4816. https://doi.org/10.1007/s00253-016-7489-5.

Valente, M., Grana, D., Malano, F., Perez, P., Quintana, C., Tirao, G., Vedelago, J., 2016. Development and characterization of a microCT facility. IEEE Latin America Transactions 14 (9), 3967-3973. https://doi.org/10.1109/TLA.2016.7785920.

Vedelago, J., Obando, D.C., Malano, F., Conejeros, R., Figueroa, R., Garcia, D., González, G., Romero, M., Santibañez, M., Strumia, M., et al., 2016. Fricke and polymer gel 2D dosimetry validation using Monte Carlo simulation. Radiat. Meas. 91, 54-64. https:// doi.org/10.1016/j.radmeas.2016.05.003.

Venning, A., Hill, B., Brindha, S., Healy, B., Baldock, C., 2005. Investigation of the PAGAT polymer gel dosimeter using magnetic resonance imaging. Phys. Med. Biol. 50 (16), 3875. https://doi.org/10.1088/0031-9155/50/16/015.

Wolfel, A., Romero, M.R., Igarzabal, C.I.A., 2017. Post-synthesis modification of hydrogels. Total and partial rupture of crosslinks: formation of aldehyde groups and recrosslinking of cleaved hydrogels. Polymer 116, 251-260. https://doi.org/10.1016/j. polymer.2017.03.068. 